\title{
SIMILARITY OF OPERATORS AND GEOMETRY OF EIGENVECTOR BUNDLES
}

\author{
HYUN-KYOUNG KWON AND SERGEI TREIL
}

\begin{abstract}
We characterize the contractions that are similar to the backward shift in the Hardy space $H^{2}$. This characterization is given in terms of the geometry of the eigenvector bundles of the operators.
\end{abstract}

\section{CONTEnts}

Notation

0. Introduction and result

1. Preliminaries

2. From uniform equivalence of bundles to curvature condition

3. Curvature condition implies similarity

4. Connection with a result by B. Sz.-Nagy and C. Foiaş 12

5. Remark about assumption $\|T\| \leq 1 \quad 13$

References

\section{Notation}

$:=\quad$ equal by definition;

$\mathbb{C} \quad$ the complex plane;

$\mathbb{D} \quad$ the unit disk, $\mathbb{D}:=\{z \in \mathbb{C}:|z|<1\}$;

$\mathbb{T} \quad$ the unit circle, $\mathbb{T}:=\partial \mathbb{D}=\{z \in \mathbb{C}:|z|=1\} ;$

$\langle\cdot, \cdot\rangle_{H} \quad$ inner product on the space $\mathrm{H}$;

$\Delta \quad$ Normalized Laplacian, $\Delta=\bar{\partial} \partial=\partial \bar{\partial}=\frac{1}{4}\left(\frac{\partial^{2}}{\partial x^{2}}+\frac{\partial^{2}}{\partial y^{2}}\right) ;$

$\mathfrak{S}_{2} \quad$ Hilbert-Schmidt class of operators;

2000 Mathematics Subject Classification. Primary 47A99, Secondary 47B32, 30D55, $53 \mathrm{C} 55$.

The work of S. Treil was supported by the National Science Foundation under Grant DMS-0501065. 
$\|\cdot\|, \mathbf{I} \cdot \mathbf{I}$ norm; since we are dealing with matrix- and operator-valued functions, we will use the symbol $\|$. $\|$ (usually with a subscript) for the norm in a function space, while $\mid$.| is used for the norm in the underlying vector (operator) space. Thus, for a vectorvalued function $f$ the symbol $\|f\|_{2}$ denotes its $L^{2}$-norm, but the symbol $|f|$ stands for the scalar-valued function whose value at a point $z$ is the norm of the vector $f(z)$;

$H^{2}, H^{\infty} \quad$ Hardy classes of analytic functions,

$$
H^{p}:=\left\{f \in L^{p}(\mathbb{T}): \hat{f}(k):=\int_{\mathbb{T}} f(z) z^{-k} \frac{|d z|}{2 \pi}=0 \text { for } k<0\right\} .
$$

Hardy classes can be identified with the spaces of functions that are analytic in the unit disk $\mathbb{D}$ : in particular, $H^{\infty}$ is the space of all functions bounded and analytic in $\mathbb{D}$;

$H_{E}^{2} \quad$ vector-valued Hardy class $H^{2}$ with values in $E$;

$H_{n}^{2} \quad$ vector-valued Hardy class $H^{2}$ with values in $\mathbb{C}^{n}$;

$L_{E_{*} \rightarrow E}^{\infty} \quad$ class of bounded functions on the unit circle $\mathbb{T}$ whose values are bounded operators from $E_{*}$ to $E$;

$H_{E_{*} \rightarrow E}^{\infty} \quad$ operator Hardy class of bounded analytic functions whose values are bounded operators from $E_{*}$ to $E$ :

$$
\|F\|_{\infty}:=\sup _{z \in \mathbb{D}}|F(z)|=\operatorname{esssup}_{\xi \in \mathbb{T}}|F(\xi)|
$$

$T_{\Phi} \quad$ Toeplitz operator with symbol $\Phi$; and

$\operatorname{tr} A \quad$ trace of the operator A.

Throughout the paper all Hilbert spaces are assumed to be separable. We always assume that in any Hilbert space, an orthonormal basis is fixed so that any operator $A: E \rightarrow E_{*}$ can be identified with its matrix. Thus, besides the usual involution $A \mapsto A^{*}$ ( $A^{*}$ is the Hilbert-adjoint of $A$ ), we have two more: $A \mapsto A^{T}$ (transpose of the matrix) and $A \mapsto \bar{A}$ (complex conjugation of the matrix), so $A^{*}=(\bar{A})^{T}=\overline{A^{T}}$. Although everything in the paper can be presented in an invariant, "coordinate-free" form, the use of transposition and complex conjugation makes the notation easier and more transparent.

\section{INTRODUCTION AND RESULT}

The main objects of this paper are operators with complete analytic families of eigenvectors, the backward shift being one of the simplest examples of such operators. Classification of such operators up to unitary equivalence was completely done by M. J. Cowen and R. G. Douglas in [4]. They had shown, in particular, that if the eigenvector bundles of such operators are equivalent as Hermitian holomorphic vector bundles, then they are unitarily 
equivalent. They had also introduced numerous local criteria of the equivalence of the eigenvector bundles (and so the unitary equivalence of the corresponding operators).

We are interested in the problem of classification of such operators up to similarity. Let us recall that operators $T_{1}$ and $T_{2}$ are similar if there exists a (bounded) invertible operator $A$ such that $T_{1}=A T_{2} A^{-1}$. It was shown already in [4 that this problem is significantly more complicated than unitary classification; in particular, it was shown in 4 that similarity of operators cannot be expressed as a local condition on their eigenvector bundles. So, we restrict ourselves to a particular case of the general problem. Namely, we are interested in the case when an operator is similar to the backward shift $S^{*}$ in the Hardy space $H^{2}$ (scalar or vector valued).

Let us recall that the backward shift $S^{*}$ in the Hardy space $H_{E}^{2}$ is the adjoint of the forward shift $S, S f(z)=z f(z), f \in H^{2}$, and can be expressed as $S^{*} f(z)=(f(z)-f(0)) / z, f \in H^{2}$. The same formulas can be used to define $S^{*}$ on the vector Hardy space $H_{E}^{2}$. Sometimes, to emphasize that we are considering $S^{*}$ in the vector Hardy class $H_{E}^{2}$ we will use the notation $S_{E}^{*}$ (or $S_{n}^{*}$ if $\operatorname{dim} E=n$ ).

Eigenvectors of $S^{*}$ are well known. Namely, the point spectrum (the set of eigenvalues) of $S^{*}$ is the open unit disc $\mathbb{D}$, and $S^{*} f=\lambda f, f \in H_{E}^{2},|\lambda|<1$, if and only if $f$ can be represented as

$$
k_{\bar{\lambda}} e, \quad e \in E \text {; }
$$

here $k_{\lambda}$ denotes the reproducing kernel 11 of the (scalar) Hardy space $H^{2}$, $k_{\lambda}(z):=1 /(1-\bar{\lambda} z)$. Probably the easiest way to see that is to represent $k_{\bar{\lambda}} e$ as the geometric series, $k_{\bar{\lambda}} e=\sum_{0}^{\infty} \lambda^{k} z^{k} e$.

We will also assume that the operator $T$ is contractive, i.e., that $\|T\| \leq 1$. As one can see from our result, one cannot expect a simple solution for the general case.

One can say that for this case the problem was solved by B. Sz.-Nagy and C. Foiaş [9, Chap 9.1], [10, [11] who proved that an operator $A,\|A\| \leq 1$, in a separable Hilbert space is similar to an isometry if and only if its characteristic function is left invertible in (operator-valued) $H^{\infty}$. Under our assumptions about $T$ the operator $T^{*}$ is completely non-unitary, so the similarity of $T^{*}$ to an isometry is equivalent to the similarity to the forward shift $S, S f(z)=z f, f \in H_{E}^{2}$, in some (generally vector-valued) $H^{2}$ space. Taking the adjoint operators, we obtain that $T$ is similar to the backward shift $S^{*}$ in some vector-valyed space $H^{2}$ if and only if the characteristic function of the operator $T^{*}$ is left invertible in $H^{\infty}$.

However, we are interested in the description only in terms of the geometry of the eigenvector bundle, and the result of B. Sz.-Nagy and C. Foiaş does not give such a description.

\footnotetext{
$1_{\text {The function }} k_{\lambda}$ is called the reproducing kernel because $\left(f, k_{\lambda}\right)=f(\lambda)$ for $f \in H^{2}$. This property explains why the notation $k_{\lambda}$ and not $k_{\bar{\lambda}}$ is used for the function $1 /(1-\bar{\lambda} z)$.
} 
We assume the following about our linear operator $T$ on a Hilbert space $H$ :

(1) $T$ is contractive, i.e., $\|T\| \leq 1$;

(2) $\operatorname{dim} \operatorname{ker}(T-\lambda I)$ is constant for all $\lambda \in \mathbb{D}$;

(3) $\operatorname{span}\{\operatorname{ker}(T-\lambda I): \lambda \in \mathbb{D}\}=H$; and

(4) the subspaces $\mathcal{E}(\lambda)=\operatorname{ker}(T-\lambda I)$ form a depend analytically on the spectral parameter $\lambda \in \mathbb{D}$.

Assumption (4) means that for each $\omega \in \mathbb{D}$ there exists a neighborhood $U_{\omega}$ of $\omega$ and a left invertible in $L^{\infty}$ analytic operator-valued function $F_{\omega}$ defined on $U_{\omega}, F_{\omega}(\lambda): E_{*} \rightarrow E$, such that $\operatorname{ran} F_{\omega}(\lambda)=\mathcal{E}(\lambda)$. It is easy to see that $\operatorname{dim} E_{*}$ must be the same for all $\omega$, so $\operatorname{dim} \mathcal{E}(\lambda)=\operatorname{dim} E_{*}$ for all $\lambda \in \mathbb{D}$, so condition (2) is redundant.

If $\operatorname{dim} E_{*}<\infty$, then the columns of $F(\lambda)$ form a basis in $\mathcal{E}(\lambda)$, so the disjoint union $\coprod_{\lambda \in \mathbb{D}} \mathcal{E}(\lambda)=\left\{\left(\lambda, v_{\lambda}\right): \lambda \in \mathbb{D}, v_{\lambda} \in \mathcal{E}(\lambda)\right\}$ is a holomorphic vector bundle over $\mathbb{D}$ (subbundle of the trivial bundle $\mathbb{D} \times H$ ) with the natural projection $\pi, \pi\left(\lambda, v_{\lambda}\right)=\lambda$.

In the case $\operatorname{dim} E_{*}=\infty$, the above statement can be used as a definition of a holomorphic vector bundle of infinite rank.

We will follow the usual agreements and write $v_{\lambda}$ instead of $\left(\lambda, v_{\lambda}\right)$, which simplifies the notation. Note, that the subspaces $\mathcal{E}(\lambda)$ inherit the metric from the Hilbert space $H$, so our bundle $\coprod_{\lambda \in \mathbb{D}} \mathcal{E}(\lambda)$ is a Hermitian holomorphic vector bundle.

One can state some assumptions about the operator $T$ that guarantee that condition (4) holds. For example, it is proven in [4] that for a bounded linear operator $T: H \rightarrow H$ such that for all $\lambda \in \mathbb{D}$ the operator $T-\lambda I$ is Fredholdm, $\operatorname{ran}(T-\lambda I)=H$ and $\operatorname{dim} \operatorname{ker}(T-\lambda I) \equiv$ const, condition (4) holds.

In order to state the result of the paper, we define on the unit disk $\mathbb{D}$ a projection-valued function $\Pi: \mathbb{D} \rightarrow B(H)$ that assigns to each $\lambda \in \mathbb{D}$, the orthogonal projection onto $\operatorname{ker}(T-\lambda I)$;

$$
\Pi(\lambda):=P_{\operatorname{ker}(T-\lambda I)} .
$$

This function is clearly $C^{\infty}$ and even real analytic in the operator norm topology, but in what follows we will only need the fact that it is a $\mathcal{C}^{2}$ function, i.e., a function twice continuously differentiable (in the operator norm topology).

Let us also recall that if $\mathcal{E}$ and $\widetilde{\mathcal{E}}$ are two holomorphic vector bundles over the same set $\Omega$, then a map $\Psi: \mathcal{E} \rightarrow \widetilde{\mathcal{E}}$ is called a bundle map if it is holomorphic, and for each $\lambda \in \Omega$ the restriction of $\Psi$ onto the fiber $\mathcal{E}(\lambda):=\pi^{-1}(\lambda)$ is a linear transformation from $\mathcal{E}(\lambda)$ to $\widetilde{\mathcal{E}}(\lambda)=\widetilde{\pi}^{-1}(\lambda)$.

Now we are ready to state our main result:

Theorem 0.1. Let $T$ be a linear operator on a Hilbert space $H$ under assumptions (1) through (4) such that $\operatorname{dim} \operatorname{ker}(T-\lambda I)=n<\infty$ for every 
$\lambda \in \mathbb{D}$. Let $\Pi(\lambda)$ be the orthogonal projection onto $\operatorname{ker}(T-\lambda I)$. Then the following statements are equivalent:

(1) $T$ is similar to the backward shift operator $S_{n}^{*}$ on $H_{n}^{2}$ via an invertible operator $A: H_{n}^{2} \rightarrow H$

(2) The eigenvector bundles of $T$ and $S_{n}^{*}$ are "uniformly equivalent". i.e., there exists a holomorphic bundle map bijection $\Psi$ from the eigenvector bundle of $S_{n}^{*}$ to that of $T$ such that for some constant $c>0$,

$$
\frac{1}{c}\left\|v_{\lambda}\right\|_{H_{n}^{2}} \leq\left\|\Psi\left(v_{\lambda}\right)\right\|_{H} \leq c\left\|v_{\lambda}\right\|_{H_{n}^{2}}
$$

for all $v_{\lambda} \in \operatorname{ker}\left(S_{n}^{*} I-\lambda\right)$ and for all $\lambda \in \mathbb{D}$;

(3) There exists a bounded subharmonic function $\varphi$ such that

$$
\Delta \varphi(z) \geq\left|\frac{\partial \Pi(z)}{\partial z}\right|_{\mathfrak{S}_{2}}^{2}-\frac{n}{\left(1-|z|^{2}\right)^{2}} \quad \text { for all } z \in \mathbb{D} .
$$

(4) The measure

$$
\left(\left|\frac{\partial \Pi(z)}{\partial z}\right|_{\mathfrak{S}_{2}}^{2}-\frac{n}{\left(1-|z|^{2}\right)^{2}}\right)(1-|z|) d x d y
$$

is Carleson and the estimate

$$
\left(\left|\frac{\partial \Pi(z)}{\partial z}\right|_{\mathfrak{S}_{2}}^{2}-\frac{n}{\left(1-|z|^{2}\right)^{2}}\right)^{\frac{1}{2}} \leq \frac{C}{1-|z|}
$$

holds.

Remark 0.2. We see in Section 1 that $\left|\frac{\partial \Pi(z)}{\partial z}\right|_{\mathfrak{S}_{2}}^{2}-\frac{n}{\left(1-|z|^{2}\right)^{2}} \geq 0$.

Remark 0.3. Treating $\operatorname{ker}(T-\lambda I)$ as a subbundle of the trivial bundle $H \times$ $\mathbb{D}$, one can see that $-\frac{\partial \Pi(\lambda)}{\partial \lambda}$ is its second fundamental form, so the mean curvature of the eigenvector bundle $\operatorname{ker}(T-\lambda)$ is $-\left|\frac{\partial \Pi(\lambda)}{\partial \lambda}\right|_{\mathfrak{S}_{2}}^{2}$. On the other hand, $-\left(1-|\lambda|^{2}\right)^{-2}=\Delta \ln \left(1-|\lambda|^{2}\right)$ is the curvature of the eigenvector bundle of $S^{*}$, so $-n\left(1-|\lambda|^{2}\right)^{-2}$ is the mean curvature of the eigenvector bundle of the backward shift $S_{n}^{*}$ of multiplicity $n$.

Thus, statements (3) and (4) are about the mean curvatures of the eigenvector bundles of $T$ and $S^{*}$.

Remark 0.4. Statement (3) of the theorem simply means that the Green potential

$$
\mathcal{G}(\lambda):=\frac{2}{\pi} \iint_{\mathbb{D}} \ln \left|\frac{z-\lambda}{1-\bar{\lambda} z}\right|\left(\left|\frac{\partial \Pi(z)}{\partial z}\right|_{\mathfrak{S}_{2}}^{2}-\frac{n}{\left(1-|z|^{2}\right)^{2}}\right) d x d y
$$

is uniformly bounded inside the unit disk $\mathbb{D}$. Integrating separately over a small neighborhood of $\lambda$ and the rest of $\mathbb{D}$, one can easily see that $(4) \Longrightarrow$ (3). 


\section{Preliminaries}

1.1. Inner-outer factorization and invariant subspaces. Let us recall that an operator-valued function $F \in H_{E_{*} \rightarrow E}^{\infty}$ is called inner if $F(z)$ is an isometry a.e. on $\mathbb{T}$, and outer if $F H_{E_{*}}^{2}$ is dense in $H_{E}^{2}$. Every $F \in H_{E_{*} \rightarrow E}^{\infty}$ can be represented as $F=F_{i} F_{o}$ for an inner function $F_{i} \in H_{E_{\#} \rightarrow E}^{\infty}$, an outer function $F_{o} \in H_{E_{*} \rightarrow E_{\#}}^{\infty}$, and an auxiliary Hilbert space $E_{\#}$.

Let $S=S_{E}$ be the (forward) shift operator on $H_{E}^{2}, S f=z f, f \in H_{E}^{2}$. The famous Beurling-Lax Theorem says that any non-zero $S$-invariant subspace $\mathcal{E} \subset H_{E}^{2}, S \mathcal{E} \subset \mathcal{E}$, can be represented as $\Theta H_{E_{*}}^{2}$, where $E_{*}$ is an auxiliary Hilbert space and $\Theta \in H_{E_{*} \rightarrow E}^{\infty}$ is an inner function. The inner function $\Theta$ is unique up to a constant unitary factor on the right.

The backward shift $S^{*}, S^{*} f=(f(z)-f(0)) / z, f \in H_{E}^{2}$, is the adjoint of $S$, so any non-trivial invariant subspace $K \subset H_{E}^{2}$ of $S^{*}$ admits the representation $K=K_{\Theta}:=H_{E}^{2} \ominus \Theta H_{E}^{2}$ with some inner function $\Theta \in H_{E_{*} \rightarrow E}^{\infty}$.

1.2. Tensor structure of the eigenvector bundle of $T$. The following theorem [8, Chap 0.2] plays a critical role in what follows.

Theorem 1.1 (Model Theorem). Every contraction $T$ on $H$ with the property that $\lim _{n}\left\|A^{n} h\right\|=0$ for every $h \in H$ is unitarily equivalent to $S_{E}^{*} \mid K$ for some Hilbert space $E$ and an $S_{E}^{*}$-invariant subspace $K$ of $H_{E}^{2}$.

For our operator $T$ we trivially have $\lim _{n}\left\|T^{n} h\right\|=0$ for linear combinations of eigenvectors, which are dense in $H$ by assumption (3). Since $T$ is a contraction, $\left\|T^{n}\right\| \leq 1$, so the standard $\varepsilon / 3$ argument shows that the conditions of Theorem 1.1. are satisfied. So, without loss of generality, we can assume that $T$ is the restriction of the backward shift $S^{*}$ in the vector Hardy space $H_{E}^{2}$ (where $E$ is an auxiliary Hilbert space) onto its invariant subspace $K \subset H_{E}^{2}$. If $K=H_{E}^{2}$ the operator $T$ is the backward shift, so we only need to consider the case when $K$ is a proper subspace of $H_{E}^{2}$. In this case $K$ can be represented as $K=K_{\Theta}$, where $\Theta \in H_{E_{*} \rightarrow E}^{\infty}$ is an inner function.

Clearly an eigenvector of $T$ is an eigenvector of $S^{*}$, and the eigenvectors of $S^{*}$ are well known. As it was shown before in the introduction the eigenspace $\operatorname{ker}\left(S^{*}-\lambda I\right)$ of the backward shift $S^{*}$ in the scalar Hardy space $H^{2}$ spanned by the reproducing kernel $k_{\bar{\lambda}}$, where recall $k_{\lambda}(z)=1 /(1-\bar{\lambda} z)$. So in the case of the backward shift $S_{E}^{*}$ in $H_{E}^{2}$,

$$
\operatorname{ker}\left(S_{E}^{*}-\lambda I\right)=\left\{k_{\bar{\lambda}}(z) e: e \in E\right\} .
$$

So, the eigenspaces of $T=S^{*} \mid K$ are given by

$$
\operatorname{ker}(T-\lambda I)=k_{\bar{\lambda}} E(\lambda)=\left\{k_{\bar{\lambda}} e: e \in E(\lambda)\right\},
$$

where the $E(\lambda)$ are some subspaces of the space $E$. The assumption (3) that $\operatorname{ker}(T-\lambda I)$ is a holomorphic vector bundle implies that the subspaces $E(\lambda)$ depend analytically on the spectral parameter $\lambda$, i.e., that the family of subspaces $E(\lambda)$ is a holomorphic vector bundle as well. 
The vector valued Hardy space $H_{E}^{2}$ is a natural realization of the tensor product $H^{2} \otimes E$, so we can write

$$
\operatorname{ker}(T-\lambda I)=k_{\bar{\lambda}} \otimes E(\lambda) .
$$

Remark 1.2. While it is not essential for the proof of the main result (Theorem 0.1$)$, it is easy to see that $E(\lambda)=\operatorname{ker} \Theta(\bar{\lambda})^{*}$, where $\Theta \in H_{E_{*} \rightarrow E}^{\infty}$ is the inner function such that $K=K_{\Theta}$. Indeed, an eigenvector $k_{\bar{\lambda}} e$ belongs to $K_{\Theta}$ if and only if $k_{\bar{\lambda}} e \perp \Theta H_{E_{*}}^{2}$. Using the reproducing kernel property of $k_{\lambda}$ we get that for $h \in H_{E_{*}}^{2}$,

$$
\left\langle\Theta h, k_{\bar{\lambda}} e\right\rangle_{H_{E}^{2}}=\langle\Theta(\bar{\lambda}) h(\bar{\lambda}), e\rangle_{E}=\left\langle h(\bar{\lambda}), \Theta(\bar{\lambda})^{*} e\right\rangle_{E} .
$$

Since $\left\{h(\bar{\lambda}): h \in H_{E_{*}}^{2}\right\}=E$, we conclude that $k_{\bar{\lambda}} e \perp \Theta H^{2}$ iff $\Theta(\bar{\lambda})^{*} e=0$. Therefore, $E(\lambda)=\operatorname{ker} \Theta(\bar{\lambda})^{*}$.

Remark 1.3. The inner function $\Theta \in H_{E_{*} \rightarrow E}^{\infty}$ appearing above is the characteristic function of the operator $T^{*}$, and therefore the spaces $E_{*}$ and $E$ can be identified with $\operatorname{clos}\left(I-T T^{*}\right)^{\frac{1}{2}} H$ and $\operatorname{clos}\left(I-T^{*} T\right)^{\frac{1}{2}} H$, respectively [9, Chap 6.2].

1.3. Curvature of the eigenvector bundle of $T$. Let us compute the norm $|\partial \Pi / \partial z|_{\mathfrak{S}_{2}}^{2}$, where $\Pi(z)$ is the orthogonal projection onto $\operatorname{ker}(T-$ $z I)$. As we mentioned before, this expression is the mean curvature of the eigenvector bundle of $T$.

Using the tensor structure $\operatorname{ker}(T-\lambda I)=k_{\bar{\lambda}} \otimes E(\lambda)$ one can represent $\Pi(\lambda)$ as

$$
\Pi(\lambda)=\Pi_{1}(\lambda) \otimes \Pi_{2}(\lambda),
$$

where $\Pi_{1}(\lambda)$ it the orthogonal projection in the (scalar) space $H^{2}$ onto $\operatorname{span}\left\{k_{\bar{\lambda}}\right\}$, and $\Pi_{2}(\lambda)$ is the orthogonal projection in $E$ onto $E(\lambda)$.

Lemma 1.4. In the above notation, if $\operatorname{rank} \Pi(\lambda)\left(=\operatorname{rank} \Pi_{2}(\lambda)\right)=n<\infty$, then

$$
\begin{aligned}
\left|\frac{\partial \Pi(\lambda)}{\partial \lambda}\right|_{\mathfrak{S}_{2}}^{2} & =\left|\frac{\partial \Pi_{1}(\lambda)}{\partial \lambda}\right|_{\mathfrak{S}_{2}}^{2}+\left|\frac{\partial \Pi_{2}(\lambda)}{\partial \lambda}\right|_{\mathfrak{S}_{2}}^{2} \\
& =\frac{n}{\left(1-|z|^{2}\right)^{2}}+\left|\frac{\partial \Pi_{2}(\lambda)}{\partial \lambda}\right|_{\mathfrak{S}_{2}}^{2}
\end{aligned}
$$

To prove this lemma we will need a couple of well known and simple facts.

Lemma 1.5. Let $E(\lambda), \lambda \in \mathbb{D}$, be an analytic family of subspaces (holomorphic vector bundle), and let $\Pi(\lambda)$ be the orthogonal projection onto $E(\lambda)$. Then

$$
(I-\Pi(z)) \frac{\partial \Pi(z)}{\partial z} \Pi(z)=\frac{\partial \Pi(z)}{\partial z} .
$$

This lemma is a well known fact in complex differential geometry, but for the sake of completeness we present the proof. 
Proof of Lemma 1.5. The fact that the family of subspaces $E(\lambda)$ is a holomorphic vector bundle means that locally the subspaces $E(\lambda)$ can be represented as $\operatorname{ran} F(\lambda)$ where $F$ is a left invertible analytic operator-valued function. Given such a representation one can write the formula for $\Pi$, $\Pi=F\left(F^{*} F\right)^{-1} F^{*}$. Direct computation shows that

$$
\frac{\partial \Pi(z)}{\partial z}=(I-\Pi(z)) F^{\prime}(z)\left(F(z)^{*} F(z)\right)^{-1} F(z)^{*}
$$

and the conclusion of the lemma follows immediately.

Lemma 1.6. For operators $A$ and $B$ in the Hilbert-Schmidt class $\mathfrak{S}_{2}$,

$$
\|A \otimes B\|_{\mathfrak{S}_{2}}^{2}=\|A\|_{\mathfrak{S}_{2}}^{2}\|B\|_{\mathfrak{S}_{2}}^{2} .
$$

This lemma is well known and the proof is an easy exercise, so we omit it.

Proof of Lemma 1.4. Using the product rule, we get from (1.2)

$$
\frac{\partial \Pi(\lambda)}{\partial \lambda}=\frac{\partial \Pi_{1}(\lambda)}{\partial \lambda} \otimes \Pi_{2}(\lambda)+\Pi_{1}(\lambda) \otimes \frac{\partial \Pi_{2}(\lambda)}{\partial \lambda}=: X+Y .
$$

The identity $\Pi_{2}(\lambda) \frac{\partial \Pi_{2}(\lambda)}{\partial \lambda}=0$ (see Lemma 1.5) implies that $X^{*} Y=0$. Therefore

$$
\begin{aligned}
\left.|X+Y|\right|_{\mathfrak{S}_{2}} ^{2}=\operatorname{tr} X^{*} X+\operatorname{tr} Y^{*} Y+2 \operatorname{Re} \operatorname{tr}\left(X^{*} Y\right) & =\operatorname{tr} X^{*} X+\operatorname{tr} Y^{*} Y \\
& =|X| \mathbf{I}_{\mathfrak{S}_{2}}^{2}+|Y|_{\mathfrak{S}_{2}}^{2}
\end{aligned}
$$

Applying Lemma 1.6 to each term and recalling that for an orthogonal projection $P$ we have $\left.|P|\right|_{\mathfrak{S}_{2}} ^{2}=\operatorname{rank} P$, we get that

$$
\left|\frac{\partial \Pi(\lambda)}{\partial \lambda}\right|_{\mathfrak{S}_{2}}^{2}=n\left|\frac{\partial \Pi_{1}(\lambda)}{\partial \lambda}\right|_{\mathfrak{S}_{2}}^{2}+\left|\frac{\partial \Pi_{2}(\lambda)}{\partial \lambda}\right|_{\mathfrak{S}_{2}}^{2} .
$$

The lemma is proved modulo computation of $\left|\frac{\partial \Pi_{1}(\lambda)}{\partial \lambda}\right|_{\mathfrak{S}_{2}}^{2}$, which is done in the next lemma.

Lemma 1.7. Let $\Pi_{1}(\lambda)$ be the orthogonal projection onto $\operatorname{span}\left\{k_{\bar{\lambda}}\right\}$ in $H^{2}$ (scalar valued). Then

$$
\left|\frac{\partial \Pi_{1}(\lambda)}{\partial \lambda}\right|_{\mathfrak{S}_{2}}^{2}=\left(1-|\lambda|^{2}\right)^{-2} \quad \text { for all } \lambda \in \mathbb{D} .
$$

Proof of Lemma 1.7. The proof can a simple exercise in complex differential geometry, using the fact that the quantity in question is (up to the sign) the curvature of the eigenvector bundle of $S^{*}$; see Remark 0.3 .

However, for the convenience of the reader we present a direct computation (one of the many possible).

First, recall that $k_{\lambda}$ is the reproducing kernel of $H^{2}$, i.e., $\left\langle f, k_{\lambda}\right\rangle=f(\lambda)$ for all $f \in H^{2}$. Using the reproducing kernel property of $k_{\lambda}$ we conclude that $\left\|k_{\lambda}\right\|_{2}^{2}=\left\langle k_{\lambda}, k_{\lambda}\right\rangle=\left(1-|\lambda|^{2}\right)^{-1}$. Therefore for $f \in H^{2}$,

$$
\Pi_{2}(\lambda) f=\left\|k_{\bar{\lambda}}\right\|_{2}^{-2}\left\langle f, k_{\bar{\lambda}}\right\rangle k_{\bar{\lambda}}=\left(1-|\lambda|^{2}\right) f(\bar{\lambda}) k_{\bar{\lambda}} .
$$


Taking $\frac{\partial}{\partial \lambda}$ and using the fact that $\frac{\partial f(\bar{\lambda})}{\partial \lambda}=0$ we get

$$
\frac{\partial \Pi_{2}(\lambda)}{\partial \lambda} f=f(\bar{\lambda})\left(-\bar{\lambda} k_{\bar{\lambda}}+\left(1-|\lambda|^{2}\right) \widetilde{k}_{\bar{\lambda}}\right)
$$

where

$$
\widetilde{k}_{\bar{\lambda}}(z)=\frac{\partial}{\partial \lambda} k_{\bar{\lambda}}(z)=\frac{z}{(1-\lambda z)^{2}} .
$$

Note, that for $f \in H^{2}$,

$$
\left\langle f, \widetilde{k}_{\lambda}\right\rangle=f^{\prime}(\lambda) .
$$

Using this identity one can get that

$$
\left\|\widetilde{k}_{\lambda}\right\|_{2}^{2}=\frac{1+|\lambda|^{2}}{\left(1-|\lambda|^{2}\right)^{3}}=\left\|\widetilde{k}_{\bar{\lambda}}\right\|_{2}^{2}
$$

The reproducing property for $k_{\lambda}$ implies that

$$
\left\langle\widetilde{k}_{\bar{\lambda}}, k_{\bar{\lambda}}\right\rangle=\frac{\bar{\lambda}}{\left(1-|\lambda|^{2}\right)^{2}} .
$$

Combining all together we can conclude that

$$
\left\|-\bar{\lambda} k_{\bar{\lambda}}+\left(1-|\lambda|^{2}\right) \widetilde{k}_{\bar{\lambda}}\right\|_{2}^{2}=\left(1-|\lambda|^{2}\right)^{-1} .
$$

Since $f(\bar{\lambda})=\left\langle f, k_{\bar{\lambda}}\right\rangle$, and as we discussed above $\left\|k_{\bar{\lambda}}\right\|_{2}^{2}=\left\|k_{\lambda}\right\|_{2}^{2}=(1-$ $\left.|\lambda|^{2}\right)^{-1}$

$$
\left|\frac{\partial \Pi_{1}(\lambda)}{\partial \lambda}\right|^{2}=\left(1-|\lambda|^{2}\right)^{-2}
$$

Since (see Lemma 1.5) $\frac{\partial \Pi(\lambda)}{\partial \lambda}$ is a rank one operator, its operator and HilbertSchmidt norms coincide.

\section{From Uniform EQUiVAlENCE OF BUNDLES to CURVATURE CONDITION}

In this section we are going to prove the implication $(2) \Longrightarrow(4)$.

Note that according to Lemma 1.4

$$
\left|\frac{\partial \Pi_{2}(\lambda)}{\partial \lambda}\right|_{\mathfrak{S}_{2}}^{2}=\left|\frac{\partial \Pi(\lambda)}{\partial \lambda}\right|_{\mathfrak{S}_{2}}^{2}-\frac{n}{\left(1-|z|^{2}\right)^{2}},
$$

so $\left|\frac{\partial \Pi(\lambda)}{\partial \lambda}\right|_{\mathfrak{S}_{2}}^{2}-\frac{n}{\left(1-|z|^{2}\right)^{2}}$ in statements (3) and (4) of Theorem 0.1 can be replaced by the curvature $\left|\frac{\partial \Pi_{2}(\lambda)}{\partial \lambda}\right|_{\mathfrak{S}_{2}}^{2}$.

Let $\Psi$ be the uniformly equivalent bundle map bijection, as in condition (2). A bundle map means that $\Psi$ is an analytic function of $\lambda$, maps the fiber $\operatorname{ker}\left(S_{n}^{*}-\lambda I\right)$ to the fiber $\operatorname{ker}(T-\lambda I)$ and is linear in each fiber $\operatorname{ker}\left(S_{n}^{*}-\lambda I\right)$.

It is easy to see from the descriptions of $\operatorname{ker}\left(S_{n}^{*}-\lambda I\right)$ and $\operatorname{ker}(T-\lambda I)$ that any such bundle map bijection is represented by

$$
\Psi\left(k_{\bar{\lambda}} e\right)=k_{\bar{\lambda}} \cdot F(\lambda) e, \quad \forall e \in \mathbb{C}^{n},
$$


where $F \in H_{\mathbb{C}^{n} \rightarrow E}^{\infty}$ is an operator-valued function such that

$$
\operatorname{ran} F(\lambda)=E(\lambda)\left(=\operatorname{ker} \Theta(\bar{\lambda})^{*}\right) .
$$

The "uniform equivalence" property of $\Psi$ means that

$$
c^{-1} I \leq F^{*} F \leq C I, \quad \forall z \in \mathbb{D} .
$$

Hence the orthogonal projection $\Pi_{2}(\lambda)$ from $E$ onto $E(\lambda)$ can be written down as

$$
\Pi_{2}=F\left(F^{*} F\right)^{-1} F^{*} .
$$

Differentiating we get $\frac{\partial \Pi_{2}(z)}{\partial z}=\left(I-\Pi_{2}(z)\right) F^{\prime}(z)\left(F(z)^{*} F(z)\right)^{-1} F(z)^{*}$, and taking into account (2.1) we have

$$
\left|\frac{\partial \Pi_{2}(z)}{\partial z}\right| \leq C\left|F^{\prime}(z)\right|
$$

The function $F$ takes values in the Hilbert-Schmidt class $\mathfrak{S}_{2}$ which is a Hilbert space, and for bounded analytic functions with values in a Hilbert space the estimate

$$
\left|F^{\prime}(z)\right| \leq C /(1-|z|)
$$

holds, and the measure

$$
\left|F^{\prime}(z)\right|^{2}(1-|z|) d x d y
$$

is Carleson. Combining these facts with (2.2) we conclude that the curvature condition (4) holds.

\section{Curvature CONDition implies Similarity}

As we already mentioned in the Introduction, see Remark 0.4 there, it is easy to show that condition (4) implies condition (3).

As it was already discussed in the beginning of the previous section, the expression $\left|\frac{\partial \Pi(\lambda)}{\partial \lambda}\right|_{\mathfrak{S}_{2}}^{2}-\frac{n}{\left(1-|z|^{2}\right)^{2}}$ in statements (3) and (4) of Theorem 0.1 can be replaced by $\left|\frac{\partial \Pi_{2}(\lambda)}{\partial \lambda}\right|_{\mathfrak{S}_{2}}^{2}$. So, the implication $(3) \Longrightarrow(1)$ follows from the theorem below, which holds even in the case $\operatorname{dim} E(\lambda)=\infty$.

Theorem 3.1. Let $E(\lambda), \lambda \in \mathbb{D}$, be an analytic family of subspaces of a Hilbert space $E$, and let $\Pi(\lambda)$ be the orthogonal projection onto $E(\lambda)$. Let

$$
T=S_{E}^{*} \mid K,
$$

where $K$ is the $S^{*}$-invariant subspace of $H_{E}^{2}, K:=\operatorname{span}\left\{k_{\bar{\lambda}} e: \lambda \in \mathbb{D}, e \in\right.$ $E(\lambda)\}$. Suppose that there exists a bounded, subharmonic function $\varphi$ such that

$$
\Delta \varphi(z) \geq\left|\frac{\partial \Pi_{2}(z)}{\partial z}\right|^{2} \quad \forall z \in \mathbb{D} .
$$

Then $T$ is similar to the backward shift $S_{E_{*}}^{*}$, where $E_{*}$ is an auxiliary Hilbert space and $\operatorname{dim} E_{*}=\operatorname{dim} E(\lambda)$. 
3.1. Toeplitz operators. To prove Theorem 3.1 we will need to recall some simple facts about Toeplitz operators. Let us recall that given an operatorvalued function $F \in L^{\infty} E \rightarrow E_{*}$, the Toeplitz operator $T_{F}: H_{E}^{2} \rightarrow H_{E_{*}}^{2}$ with symbol $F$ is defined by the formula

$$
T_{F} f=P_{+}(F f), \quad f \in H_{E}^{2}
$$

where $P_{+}$is the orthogonal projection in $L^{2}$ onto $H^{2}$.

If the symbol $F$ is analytic $\left(F \in H_{E \rightarrow E_{*}}^{\infty}\right)$, then the Toeplits operator $T_{F}$ is simply the multiplication operator (more precisely, its restriction onto $H^{2}$ ).

It is easy to see that if $F, G \in H^{\infty}$, then

$$
T_{F} T_{G}=T_{F G} .
$$

We will also need the following well known and easy to prove fact that in $F \in H_{E \rightarrow E_{*}}^{\infty}$, then

$$
T_{F^{*}} k_{\lambda} e=k_{\lambda} F^{*}(\lambda) e, \quad e \in E_{*}
$$

and

$$
T_{F^{*}} S_{E_{*}}^{*}=S_{E}^{*} T_{F^{*}}
$$

3.2. Proof of Theorem 3.1. We want to prove the existence of an invertible operator $A: H_{n}^{2} \rightarrow K$ satisfying the intertwining relation $A S_{n}^{*}=T A$ (recall that $T=S_{E}^{*} \mid K$ ).

We will need the following theorem by S. Treil and B. D. Wick [17].

Theorem 3.2. Let $\Pi: \mathbb{D} \rightarrow B(E)$ be a $\mathcal{C}^{2}$ function whose values are orthogonal projections in $E$, satisfying the identity $\Pi(z) \frac{\partial \Pi(z)}{\partial z}=0$. Assume that for some bounded subharmonic function $\varphi$, we have

$$
\Delta \varphi(z) \geq\left|\frac{\partial \Pi(z)}{\partial z}\right|^{2} \quad \text { for all } z \in \mathbb{D} .
$$

Then there exists a bounded analytic projection onto $\operatorname{ran} \Pi(z)$, i.e., a function $\mathcal{P} \in H_{E \rightarrow E}^{\infty}$ such that $\mathcal{P}(z)$ is a projection onto $\operatorname{ran} \Pi(z)$ for all $z \in \mathbb{D}$.

By Lemma 1.5, the function $\Pi$ from Theorem 3.1 satisfies the identity $\Pi(z) \frac{\partial \Pi(z)}{\partial z}=0$, so applying Theorem 3.2 to it we get a bounded analytic projection $\mathcal{P}(z)$ onto ran $\Pi(z)$. Consider the inner-outer factorization $\mathcal{P}=$ $\mathcal{P}_{\mathrm{i}} \mathcal{P}_{\mathrm{o}}$ of $\mathcal{P}$, where $\mathcal{P}_{\mathrm{i}} \in H_{E_{*} \rightarrow E}^{\infty}$ is the inner part and $\mathcal{P}_{\mathrm{o}} \in H_{E \rightarrow E_{*}}^{\infty}$ is the outer part of $\mathcal{P}$. Define a function $\mathcal{P}_{\mathrm{i}}^{\sharp}$ by $\mathcal{P}_{\mathrm{i}}^{\sharp}(z):=\mathcal{P}_{\mathrm{i}}(\bar{z})$, and consider the Toeplitz operator $T_{\mathcal{P}_{\mathrm{i}}^{\sharp}}$. Since $\left(\mathcal{P}_{\mathrm{i}}^{\sharp}\right)^{*} \in H_{E \rightarrow E_{*}}^{\infty}$, (3.3) implies

$$
T_{\mathcal{P}_{\mathrm{i}}^{\sharp}} S_{E_{*}}^{*}=S_{E}^{*} T_{\mathcal{P}_{\mathrm{i}}^{\sharp}}
$$

If we show that the operator $T_{\mathcal{P}_{i}^{\sharp}}$ is left invertible and that $\operatorname{ran} T_{\mathcal{P}_{i}^{\sharp}}=K$, we are done: the operator $A$ we want to find is simply the Toeplitz operator $T_{\mathcal{P}_{\mathrm{i}}^{\sharp}}$ treated as an operator $H_{E_{*}}^{2} \rightarrow K$. The left invertibility of $T_{\mathcal{P}_{\mathrm{i}}^{\sharp}}$ together with 
$\operatorname{ran} T_{\mathcal{P}_{\mathrm{i}}^{\sharp}}=K$ means that $A$ is invertible, and the intertwining $A S_{E_{*}}^{*}=T A$ follows from (3.4).

The left invertibility of $T_{\mathcal{P}_{i}^{\sharp}}$ is a corollary of the following lemma.

Lemma 3.3. The outer part $\mathcal{P}_{\mathrm{o}}$ is a left inverse of $\mathcal{P}_{\mathrm{i}}$, i.e., $\mathcal{P}_{\mathrm{o}} \mathcal{P}_{\mathrm{i}} \equiv I$ for all $z \in \mathbb{D}$.

This lemma immediately implies that $T_{\mathcal{P}_{\mathrm{O}}^{\sharp}}$, where $\mathcal{P}_{\mathrm{O}}^{\sharp}(z):=\mathcal{P}_{0}(\bar{z})$, is a left inverse of $T_{\mathcal{P}_{i}^{\sharp}}$. Indeed

$$
\left(T_{\mathcal{P}_{\mathrm{i}}^{\sharp}}\right)^{*}\left(T_{\mathcal{P}_{\mathrm{o}}^{\sharp}}\right)^{*}=T_{\left(\mathcal{P}_{\mathrm{i}}^{\sharp}\right)^{*}} T_{\left(\mathcal{P}_{\mathrm{O}}^{\sharp}\right)^{*}}=T_{\left(\mathcal{P}_{\mathrm{i}}^{\sharp}\right) *\left(\mathcal{P}_{\mathrm{o}}^{\sharp}\right)^{*}}=I .
$$

The last equality holds because $\mathcal{P}_{\mathrm{o}} \mathcal{P}_{\mathrm{i}} \equiv I$ and $T_{I}=I$; the previous one follows from (3.1) because $\left(\mathcal{P}_{\mathrm{i}}^{\sharp}\right)^{*},\left(\mathcal{P}_{\mathrm{O}}^{\sharp}\right)^{*} \in H^{\infty}$.

Proof of Lemma 3.3. It follows from (3.1) that

$$
T_{\mathcal{P}_{\mathrm{i}}} T_{\mathcal{P}_{\mathrm{o}}}=T_{\mathcal{P}}=T_{\mathcal{P}^{2}}=T_{\mathcal{P}_{\mathrm{i}} \mathcal{P}_{\mathrm{o}} \mathcal{P}_{\mathrm{i}} \mathcal{P}_{\mathrm{o}}}=T_{\mathcal{P}_{\mathrm{i}}} T_{\mathcal{P}_{\mathrm{o}} \mathcal{P}_{\mathrm{i}}} T_{\mathcal{P}_{\mathrm{o}}}
$$

The operator $T_{\mathcal{P}_{\mathrm{o}}}$ has dense range because $\mathcal{P}_{\mathrm{o}}$ is outer, and $\operatorname{ker} T_{\mathcal{P}_{\mathrm{i}}}=\{0\}$ because $\mathcal{P}_{\mathrm{i}}$ is inner (in fact, $T_{\mathcal{P}_{\mathrm{i}}}=\{0\}$ is an isometry). Therefore $T_{\mathcal{P}_{\mathrm{o}} \mathcal{P}_{\mathrm{i}}}=I$, so $\mathcal{P}_{\mathrm{o}} \mathcal{P}_{\mathrm{i}} \equiv I$ for all $z \in \mathbb{D}$.

To complete the proof of Theorem 3.1 it remains to show that $\operatorname{ran} T_{\mathcal{P}_{\mathrm{i}}^{\sharp}}=$ $K$. First of all, let us notice that

$$
\operatorname{ran} \mathcal{P}_{\mathrm{i}}(\lambda)=E(\lambda) \quad \forall \lambda \in \mathbb{D} .
$$

Indeed, the inclusion $E(\lambda)=\operatorname{ran} \mathcal{P}(\lambda) \subset \operatorname{ran} \mathcal{P}_{\mathrm{i}}(\lambda)$ is trivial because of the factorization $\mathcal{P}=\mathcal{P}_{\mathrm{i}} \mathcal{P}_{\mathrm{o}}$. Since $\mathcal{P}_{\mathrm{o}}$ is outer, the set ran $\mathcal{P}_{\mathrm{o}}(\lambda)$ is dense in $E_{*}$ for all $\lambda \in \mathbb{D}$. But $\mathcal{P}_{\mathrm{i}}(\lambda) \operatorname{ran} \mathcal{P}_{\mathrm{o}}(\lambda)=E(\lambda)$, so $\operatorname{ran} \mathcal{P}_{\mathrm{i}}(\lambda) \subset E(\lambda)$. It follows from (3.2) that

$$
T_{\mathcal{P}_{\mathrm{i}}^{\sharp}} k_{\bar{\lambda}} e=k_{\bar{\lambda}} \mathcal{P}_{\mathrm{i}}^{\sharp}(\bar{\lambda}) e=k_{\bar{\lambda}} \mathcal{P}_{\mathrm{i}}(\lambda) e .
$$

To see that we got all the complex conjugates correctly, one can fix bases in $E$ and $E_{*}$, consider the matrix representation of the operators, and notice that $\mathcal{P}^{\sharp}(z)=\left(\mathcal{P}^{T}(z)\right)^{*}$. Then a direct application of (3.2) implies (3.5).

The equality $\operatorname{ran} \mathcal{P}_{\mathrm{i}}(\lambda)=E(\lambda)$ together with (3.5) imply that

$$
T_{\mathcal{P}_{\mathrm{i}}^{\sharp}} k_{\bar{\lambda}} \otimes E_{*}=k_{\bar{\lambda}} \otimes E(\lambda) \text {. }
$$

Since $\operatorname{span}\left\{k_{\bar{\lambda}} \otimes E_{*}: \lambda \in \mathbb{D}\right\}=H_{E_{*}}^{2}, \operatorname{span}\left\{k_{\bar{\lambda}} \otimes E(\lambda): \lambda \in \mathbb{D}\right\}=K$, and the operator $T_{\mathcal{P}_{\mathrm{i}}^{\sharp}}$ is left invertible, we conclude that $\operatorname{ran} T_{\mathcal{P}_{\mathrm{i}}^{\sharp}}=K$.

\section{Connection with a Result by B. Sz.-Nagy and C. Foiaş}

We already mentioned in the Introduction the following result by B. Sz.Nagy and C. Foiaş [6, Chap 1.5], [9, Chap 9.1], [10], [11].

Theorem 4.1. A contraction $A(\|A\| \leq 1)$ in a Hilbert space is similar to an isometry if and only if its characteristic function is left invertible in $H^{\infty}$. 
We are not giving the definition of the characteristic function of a contraction here, because it is quite technical and is not relevant to our paper. The reader only needs to know that if $A=T^{*}$, where $T$ is the restriction of the backward shift $S^{*}$ onto an $S^{*}$-invariant subspace $K=K_{\Theta} \subset H_{E}^{2}$, then the inner function $\Theta$ is the characteristic function of $A$.

If the operator $A=T^{*}$ is unitarily equivalent to an isometry $U$, the isometry $U$ must be unitarily equivalent to the forward shift $S_{E_{*}}$ in $H_{E_{*}}^{2}$. Indeed, since $\lim _{n}\left\|T^{n} f\right\|=0$ for all $f \in K$, the same holds for $U^{*}, \lim _{n}\left\|\left(U^{*}\right)^{n} x\right\|=$ 0 for all $x$. But it is a well known fact (an easy corollary of the KolmogorovVold decomposition of isometries) that such isometry $U$ is unitarily equivalent to the forward shift $S$ in $H_{E_{*}}^{2}$. So, applying Theorem 4.1 to our situation we get that $T$ is similar to the backward shift if and only if the inner function $\Theta$ is left invertible in $H^{\infty}$.

We would like to investigate what the relation between this statement and our result is. The following remarkable lemma by N. Nikolski provides that necessary connection.

Lemma 4.2. Let $F \in H_{E_{*} \rightarrow E}^{\infty}$ satisfy

$$
F(z)^{*} F(z) \geq \delta^{2} I \quad \text { for all } z \in \mathbb{D} .
$$

Then $F$ is left invertible in $H^{\infty}$, i.e., there exists a $G \in H_{E \rightarrow E_{*}}^{\infty}$ such that $G F \equiv I$, if and only if there exists a function $\mathcal{P} \in H_{E \rightarrow E}^{\infty}$ whose values are projections (not necessarily orthogonal) onto $\operatorname{ran} F(z)$ for all $z \in \mathbb{D}$.

By this lemma, $T$ is similar to a backward shift if and only if there exists a bounded analytic projection $\mathcal{P}(z)$ onto $\operatorname{ran} \Theta(z)$. Let $\mathcal{Q}=I-\mathcal{P}$ be the complementary projection. Then $\mathcal{Q}(z)^{*}$ is a projection onto $(\operatorname{ran} \Theta(z))^{\perp}=$ $\operatorname{ker} \Theta(z)^{*}$. But as we discussed above in Section 1.2, $\operatorname{ker} \Theta(z)^{*}=E(\bar{z})$.

Notice, that the function $z \mapsto \mathcal{Q}(z)^{*}$ is antianalytic, so $T$ is similar to the backward shift iff there exists a bounded antianalytic projection onto ker $\Theta(z)^{*}$, or equivalently, a bounded analytic projection onto $E(z)=$ $\operatorname{ker} \Theta(\bar{z})^{*}$.

Of course this is only a sketch and we leave all the details to the reader as an exercise.

\section{REMARK ABOUt ASSUMption $\|T\| \leq 1$}

In this section we will show that the assumption $\|T\| \leq 1$ is essential for Theorem 0.1 . We will show that if we omit this assumption, it is possible to construct an operator $T$ whose eigenvector bundle is uniformly equivalent to that of $S^{*}$ (in the scalar Hardy space), but such that $T$ ans $S^{*}$ are not even quazisimilar.

Let us recall that operators $T_{1}$ and $T_{2}$ are called quazisimilar if there exist operators $A$ and $B$ with dense ranges and trivial kernels such that

$$
A T_{1}=T_{2} A, \quad T_{1} B=B T_{2}
$$

(if $T_{1}$ and $T_{2}$ are similar, then $B=A A^{-1}$ ). 
Theorem 5.1. Given $\varepsilon>0$, there exists an operator $T$ such that

(1) $T$ satisfies assumptions (2) through (4) from the introduction with dim $\operatorname{ker}(T-\lambda I)=1$ for $\lambda \in \mathbb{D}$;

(2) $T^{*}$ is "almost isometry", i.e.,

$$
(1+\varepsilon)^{-1}\|x\| \leq\left\|T^{*} x\right\| \leq(1+\varepsilon)\|x\| \quad \forall x \in H
$$

(3) the eigenvector bundles of $T$ and $S^{*}$ are almost isomerically equivalent, i.e., there exists a bundle map bijection $\Psi$ from the eigenvector bundle of $S^{*}$ to that of $T$ such that

$$
(1+\varepsilon)^{-1}\left\|v_{\lambda}\right\| \leq\left\|\Psi\left(v_{\lambda}\right)\right\| \leq(1+\varepsilon)\left\|v_{\lambda}\right\|
$$

for all $\lambda \in \mathbb{D}$ and for all $v_{\lambda} \in \operatorname{ker}\left(S^{*}-\lambda I\right)$;

and such that the only operator $A$ satisfying $A T=S^{*} A$ is $A=0$, so $A$ is not even quazisimilar to $S^{*}$.

We will construct the operator $T$ as the backward shift, i.e., the adjoint of the forward shift $\mathbf{S}$ in the space $H_{w}^{2}$, with the weight sequence $w=\left\{w_{k}\right\}_{1}^{\infty}$ $\left(w_{k}>0\right)$ :

$$
H_{w}^{2}:=\left\{f=\sum_{n \geq 0} a_{n} z^{n}:\|f\|_{w}^{2}:=\sum_{n \geq 0}\left|a_{n}\right|^{2} w_{n}<\infty\right\} .
$$

If one assumes that $\liminf \inf _{n}\left|a_{n}\right|^{1 / n}=1$, the space $H_{w}^{2}$ is a space of analytic in the unit disc $\mathbb{D}$ functions. Moreover, for all $\lambda \in \mathbb{D}$ the functional $f \mapsto f(\lambda)$ is bounded, so for each $\lambda \in \mathbb{D}$ there exists a unique function $\mathbf{k}_{\lambda} \in H_{w}^{2}$ (the reproducing kernel of $H_{w}^{2}$ ) such that

$$
\left\langle f, \mathbf{k}_{\lambda}\right\rangle=f(\lambda) \quad \forall f \in H_{w}^{2} .
$$

The reproducing kernel $\mathbf{k}_{\lambda}$ can be easily computed. Namely, it is easy to see that if $\left\{\varphi_{n}\right\}_{0}^{\infty}$ is an orthonormal basis in $H_{w}^{2}$, then

$$
\mathbf{k}_{\lambda}(z)=\sum_{0}^{\infty} \overline{\varphi_{n}(\lambda)} \varphi_{n}(z) .
$$

Taking the orthonormal basis $\left\{z^{n} / \sqrt{w_{n}}\right\}_{0}^{\infty}$, we get

$$
\mathbf{k}_{\lambda}(z)=\sum_{n=0}^{\infty} \frac{z^{n}}{w_{n}} .
$$

Note that the Hardy space $H^{2}$ is a particular case $\left(w_{n}=1\right.$ for all $\left.n\right)$ of the space $H_{w}^{2}$, and formula (5.2) in this case gives the reproducing kernel $k_{\lambda}(z)=1 /(1-\bar{\lambda} z)$ of $H^{2}$.

If one assumes that $\sup _{n} w_{n+1} / w_{n}<\infty$, then the shift operator $\mathbf{S}$, $\mathbf{S} f(z)=z f(z)$ is a bounded operator in $H_{w}^{2}$. The adjoint $\mathbf{S}^{*}$ is called the backward shift, and it is easy to see that

$$
\mathbf{S}^{*}\left(\sum_{n=0}^{\infty} a_{n} z^{n}\right)=\sum_{n=0}^{\infty} \frac{w_{n+1}}{w_{n}} a_{n+1} z^{n} .
$$


From this formula and (5.2) one easily concludes that

$$
\operatorname{ker}\left(\mathbf{S}^{*}-\lambda I\right)=\operatorname{span}\left\{\mathbf{k}_{\bar{\lambda}}\right\} .
$$

It follows from the reproducing kernel property that $\operatorname{span}\left\{\mathbf{k}_{\lambda}: \lambda \in \mathbb{D}\right\}=$ $H_{w}^{2}$, so $T=\mathbf{S}^{*}$ satisfies the assumptions (1)-(4) from the Introduction.

Condition (2) of Theorem 5.1 is satisfied if and only if

$$
(1+\varepsilon)^{-2} \leq w_{n+1} / w_{n} \leq(1+\varepsilon)^{2} \quad \forall n \geq 0 .
$$

The mapping $\Psi, \Psi\left(a k_{\lambda}\right)=a \mathbf{k}_{\lambda}, a \in \mathbb{C}, \lambda \in \mathbb{D}$, is clearly a holomorphic bundle map bijection between the eigenvector bundles of $S^{*}$ and $\mathbf{S}^{*}$. Since $\left\|k_{\lambda}\right\|_{H^{2}}^{2}=\left\langle k_{\lambda}, k_{\lambda}\right\rangle=k_{\lambda}(\lambda)\left(=\left(1-|\lambda|^{2}\right)^{-1}\right)$ and similarly $\left\|\mathbf{k}_{\lambda}\right\|_{H_{w}^{2}}^{2}=\mathbf{k}_{\lambda}(\lambda)$, condition (3) of Theorem 5.1 is equivalent to the estimate

$$
(1+\varepsilon)^{-2} k_{\lambda}(\lambda) \leq \mathbf{k}_{\lambda}(\lambda) \leq(1+\varepsilon)^{2} k_{\lambda}(\lambda) \quad \forall \lambda \in \mathbb{D} .
$$

Lemma 5.2. If $\sup _{n} w_{n}=\infty$, then there is no non-zero bounded operator $A$ satisfying $A \mathbf{S}^{*}=S A$.

Proof. Let $A \mathbf{S}^{*}=S^{*} A$ for some $A \neq 0$. Then $\mathbf{S} A^{*}=A^{*} S$ and therefore $\mathbf{S}^{n} A^{*}=A^{*} S^{n}$

Take $f \in H^{2}$ such that $A^{*} f=\sum_{0}^{\infty} a_{n} z^{n} \neq 0$. Pick $m$ such that $a_{m} \neq 0$. Then

$$
\left\|\mathbf{S}^{n} A f\right\|^{2}=\sum_{j=0}^{\infty}\left|a_{j}\right|^{2} w_{j+n} \geq\left|a_{m}\right|^{2} w_{m+n},
$$

so $\sup _{n}\left\|\mathbf{S}^{n} A f\right\|=\infty$ because $\sup _{n} w_{m+n}=\infty$.

On the other hand, $\left\|A^{*} S^{n} f\right\|_{H_{w}^{2}} \leq\left\|A^{*}\right\|\|f\|_{H^{2}}$, giving us a contradiction.

So, to prove the theorem, we need to find an unbounded sequence $\left\{w_{n}\right\}_{1}^{\infty}$ satisfying (5.3), and such that (5.4) holds. We define the sequence $\left\{w_{n}\right\}_{1}^{\infty}$ to be 1 for all $n$ except in sparse intervals from $N_{j}$ to $N_{j}+2 j$; the numbers $N_{j}$ will be specified later. On the intervals $\left[N_{j}, N_{j}+2 j\right]$ the sequence has "spikes": $\ln w_{n}$ on $\left[N_{j}, N_{j}+2 j\right]$ is the piecewise affine function with slope $\pm 2 \ln (1+\varepsilon)$, increasing from 0 to $2 j \ln (1+\varepsilon)$ on $\left[N_{j}, N_{j}+j\right]$ and decreasing back to 0 on $\left[N_{j}+j, N_{j}+2 j\right]$; see Figure 1.

Formally, we write

$$
\ln w_{n}= \begin{cases}2 m \ln (1+\epsilon) & n=N_{k}+m, 0 \leq m \leq j \\ 2(j-m) \ln (1+\epsilon) & n=N_{k}+j+m, 0 \leq m \leq j \\ 0 & \text { otherwise }\end{cases}
$$

We pick a sequence $N_{j}$ such that

$$
\begin{aligned}
& N_{j}+2 j<N_{j+1} \\
& \frac{2 j-1}{N_{j}+2 j} \leq \frac{\alpha}{2^{j}},
\end{aligned}
$$

where $\alpha$ is a small number such that $1-\alpha \geq(1+\varepsilon)^{-2}$. 


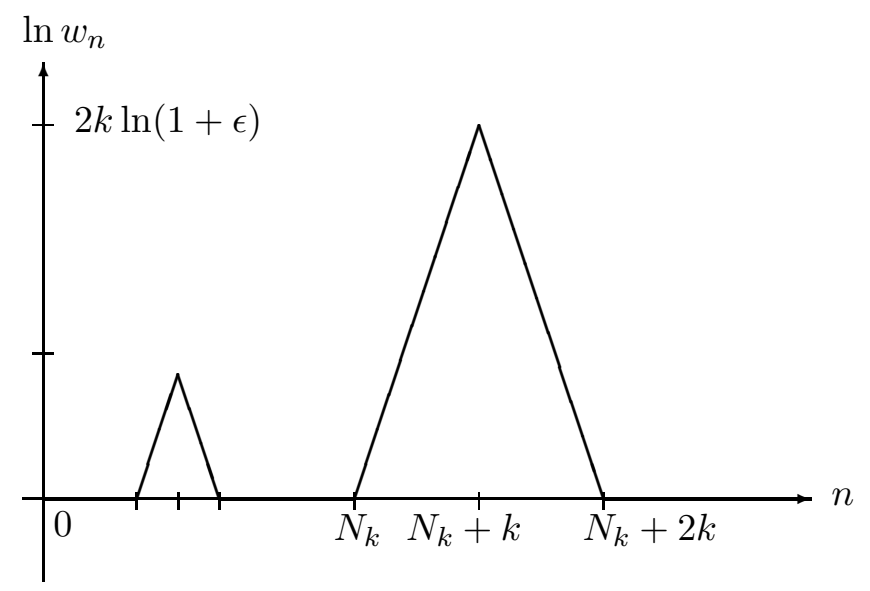

Figure 1. The function $\ln w_{n}$ : two "spikes" are shown

The sequence $\left\{w_{n}\right\}_{0}^{\infty}$ is clearly unbounded. Because of the slope condition for $\ln w_{n}$ condition (5.3) is satisfied.

The Lemma below completes the proof of the theorem.

Lemma 5.3. For the weight $w=\left\{w_{n}\right\}_{0}^{\infty}$ constructed above, the reproducing kernel $\mathbf{k}_{\lambda}$ of $H_{w}^{2}$ satisfies the inequality (5.4).

Proof of Lemma 5.3. Since $w_{n} \geq 1$,

$$
\mathbf{k}_{\lambda}(\lambda)=\sum_{n \geq 0} \frac{1}{w_{n}}|\lambda|^{2 n} \leq \sum_{n \geq 0}|\lambda|^{2 n}=k_{\lambda}(\lambda) \leq(1+\varepsilon)^{2} k_{\lambda}(\lambda),
$$

so one estimate is obvious. To get the other one, it is enough to show that $k_{\lambda}(\lambda)-\mathbf{k}_{\lambda}(\lambda) \leq \alpha k_{\lambda}(\lambda)$.

Since $1-1 / w_{n} \neq 0$ only for $n \in\left(N_{j}, N_{j}+2 j\right)$, we can write

$$
k_{\lambda}(\lambda)-\mathbf{k}_{\lambda}(\lambda)=\sum_{n \geq 0}\left(1-\frac{1}{w_{n}}\right)|\lambda|^{2 n}=\sum_{j=1}^{\infty} \sum_{n=N_{j}+1}^{N_{1}+2 j-1}\left(1-\frac{1}{w_{n}}\right)|\lambda|^{2 n} .
$$

For each $j$

$$
\begin{aligned}
\sum_{n=N_{j}+1}^{N_{j}+2 j-1}\left(1-\frac{1}{w_{n}}\right)|\lambda|^{2 n} & \leq \sum_{n=N_{j}+1}^{N_{j}+2 j-1}|\lambda|^{2 n} \\
& =\frac{|\lambda|^{2\left(N_{j}+1\right)}-|\lambda|^{2\left(N_{j}+2 j\right)}}{1-|\lambda|^{2}} \leq \frac{A_{j}}{1-|\lambda|^{2}}
\end{aligned}
$$

where

$$
A_{j}=\max \left\{x^{N_{j}+1}-x^{N_{j}+2 j}: 0 \leq x \leq 1\right\}
$$


(here $x=|\lambda|^{2}$ ). The maximum is attained at $x=\left(\frac{N_{j}+1}{N_{k}+2 j}\right)^{\frac{1}{2 j-1}}$, and

$$
A_{j}=\left(\frac{N_{j}+1}{N_{j}+2 j}\right)^{\frac{N_{j}+1}{2 j-1}} \frac{2 j-1}{N_{j}+2 j} \leq \frac{2 j-1}{N_{j}+2 j} \leq \frac{\alpha}{2^{j}}
$$

by our choice of $N_{j}$.

Summing over $j$ we get

$$
k_{\lambda}(\lambda)-\mathbf{k}_{\lambda}(\lambda) \leq \frac{1}{1-|\lambda|^{2}} \sum_{j=1}^{\infty} \alpha 2^{-j}=\frac{\alpha}{1-|\lambda|^{2}}=\alpha k_{\lambda}(\lambda),
$$

so the lemma is proved.

\section{REFERENCES}

[1] M. Andersson, The Corona Theorem for Matrices, Math. Z., 201 (1989), 121-130.

[2] J. Aubin, Applied Functional Analysis, John Wiley \& Sons, Inc., New York, 1979, Exercises by Bernard Cornet and Jean-Michel Lasry, Translated by Carole Labrousse.

[3] L. Carleson, Interpolations by bounded analytic functions and the Corona problem, Ann. of Math. (2) 76, (1962), 547-559.

[4] M. J. Cowen and R. G. Douglas, Complex Geometry and Operator Theory, Acta. Math. 141 (1978), 187-261.

[5] P. Griffiths and J. Harris, Principles of Algebraic Geometry, John Wiley \& Sons, Inc., New York, 1994.

[6] N. K. Nikolski, Operators, Functions, and Systems: An Easy Reading. Vol. 1: Hardy, Hankel, and Toeplitz, Mathematical Surveys and Monographs, Vol. 92, American Mathematical Society, Providence, RI, 2002, Translated from the French by Andreas Hartmann.

[7] _ Operators, Functions, and Systems: An Easy Reading. Vol. 2: Model Operators and Systems, Mathematical Surveys and Monographs, Vol. 93, American Mathematical Society, Providence, RI, 2002, Translated from the French by Andreas Hartmann.

[8] _ Treatise on the Shift Operator, Grundlehren der Mathematischen Wissenschaften [Fundamental Principles of Mathematical Sciences], vol. 273, SpringerVerlag, Berlin, 1986, Spectral function theory, With an appendix by S. V. Hruščev [S. V. Khrushchëv] and V. V. Peller, Translated from the Russian by Jaak Peetre.

[9] B. Sz.-Nagy and C. Foiaş, Harmonic Analysis of Operators on Hilbert Space, NorthHolland Publishing Co., Amsterdam, 1970, Translated from the French and revised.

[10] B. Sz.-Nagy and C. Foias,, On the structure of intertwining operators, Acta Sci. Math. Szeged, 35 (1973), 225-254.

[11] B. Sz.-Nagy and C. Foiaş, Sur les contractions de l'espace de Hilbert. X. Contractions similaires à des transformations unitaires, Acta Sci. Math. Szeged, 26 (1965), 79-91.

[12] S. R. Treil, Angles between co-invariant subspaces, and the operator corona problem. The Szökefalvi-Nagy problem, Dokl. Akad. Nauk SSSR 302 (1988), No. 5, 1063-1068.

[13] - Geometric methods in spectral theory of vector-valued functions: some recent results, Toeplitz operators and spectral function theory, Oper. Theory Adv. Appl., Vol. 42, Birkhäuser, Basel, 1989, pp. 209-280.

[14] Unconditional bases of invariant subspaces of a contraction with finite defects, Indiana Univ. Math. J. 46 (1997), No. 4, 1021-1054.

[15] _ An operator Corona Theorem, Indiana Univ. Math. J. 53 (2004), No. 6, 1765-1784. 
[16] Lower bounds in the matrix corona theorem and the codimension one conjecture, Geometric and Functional Analysis, 14 (2004), 1118-1133.

[17] S. Treil and B. D. Wick, Analytic Projections, Corona Problem and Geometry of Holomorphic Vector Bundles, arXiv:math/0702756 1 , accepted to the Journal of American Mathematical Society.

[18] A. Uchiyama, Corona theorems for countably many functions and estimates for their solutions, preprint, UCLA, 1980.

Department of Mathematics, Brown University, 151 Thayer Street Box 1917, PROVIDENCE, RI USA 02912 